\title{
A Novel Flocked Swab Protocol Proves to Be an Effective Method for Culturing Elevator-Containing Endoscopes
}

\author{
Jennifer Y. Lui ${ }^{1} \oplus$ - Christopher G. Chapman ${ }^{1} \cdot$ Irving Waxman $^{1} \cdot$ Uzma D. Siddiqui ${ }^{1}$
}

Received: 4 November 2020 / Accepted: 26 February 2021 / Published online: 13 March 2021

(c) The Author(s), under exclusive licence to Springer Science+Business Media, LLC, part of Springer Nature 2021

\begin{abstract}
Background The challenging disinfection process for the elevator mechanism on duodenoscopes and linear echoendoscopes has been identified as a source of clinically significant bacterial transmission. Despite increased awareness, there continues to be a lack of definitive guidelines for bacterial culturing protocols for elevator-containing endoscopes.

Aims To compare two different prospective bacterial surveillance protocols for duodenoscopes and linear echoendoscopes with regard to accuracy, efficiency, and cost.

Methods Consecutive duodenoscopes and linear echoendoscopes used at a single tertiary care center were reprocessed following hospital and manufacturer guidelines, dried using an automatic endoscope-drying machine, and hung overnight in an upright position. Following reprocessing, culture samples were sequentially obtained from each endoscope using two methods, first, the brush protocol followed immediately by the swab protocol.

Results A total of 532 primary cultures were collected from 17 duodenoscopes and eight linear echoendoscopes. Of these, 266 cultures gathered using the brush protocol were negative, while 266 cultures gathered using the swab protocol resulted in three positive cultures (1.1\%). Positive cultures showed Enterobacter cloacae and Klebsiella pneumoniae from one duodenoscope and two linear echoendoscopes. Yearly, the brush protocol amounts to approximately 520 nursing hours, and the swab protocol takes an estimated 42 nursing hours. Annually, the swab protocol could save over $\$ 26,500$ and 478 nursing hours. Conclusions The proposed swab protocol was superior to the brush protocol when evaluating the presence of residual bacteria on elevator-containing endoscopes following reprocessing and saves cost and nursing hours.
\end{abstract}

Keywords Duodenoscope $\cdot$ Linear echoendoscope $\cdot$ Elevator $\cdot$ Bacterial surveillance

\section{Introduction Background}

In recent years, transmission of residual bacteria from sideviewing duodenoscopes has resulted in fatal consequences for some patients undergoing endoscopic retrograde cholangiopancreatography (ERCP) [1]. Despite following hospital and manufacturer guidelines for endoscope reprocessing, residual bacterial contamination continues to be a problem for endoscopes with an elevator mechanism. In addition to duodenoscopes, our group previously demonstrated that this issue also applies to linear echoendoscopes used for endoscopic ultrasound (EUS) and remains a potential etiologic source of scope-to-patient bacterial transmission [2]. As

Jennifer Y. Lui

Jennifer.Lui@uchospitals.edu

1 Center for Endoscopic Research and Therapeutics, UChicago Medicine, Chicago, IL, USA a result of these fatal incidents, increased focus has been drawn to the development of endoscopic bacterial surveillance protocols for these specific types of endoscopes. Prospective bacterial surveillance provides a method to detect residual bacteria on elevator-containing endoscopes in an effort to prevent endoscope-related bacterial outbreaks.

Currently, the Centers for Disease Control and Prevention have presented recommendations of sampling and culturing protocols for duodenoscopes, but there continues to be a lack of definitive guidelines for prospective bacterial culturing protocols for both duodenoscopes and linear echoendoscopes [3]. Over the past five years, our institution has conducted post-high-level disinfection bacterial surveillance of duodenoscope and linear echoendoscope using a multistep brush protocol that was developed by the infection control department [2]. However, there are limitations to the brush protocol including the risk of inadequate sampling of deep crevices of the elevator and for environmental or 
cross-contamination from other parts of the endoscope [4]. Conducting focused testing on the elevator mechanism in mid-position ensures sampling of the small crevices surrounding the mechanism and theoretically could reduce the risk of sample contamination. Research has also shown that a flocked swab is more effective compared to a cleaning brush for detecting microbial growth [5]. The increased bioabsorbent properties of the flocked swab allow it to better capture bacteria and reach the small crevices of the elevator mechanism. Protocols for detecting the presence of residual bacteria are critical to patient safety and preventing endoscope-related bacterial outbreaks; however, the protocols must be continuously evaluated and optimized for effectiveness as well as efficiency.

The proposed novel swab protocol could offer a more accurate, efficient, and cost-saving method to identify contaminated endoscopes. The aim of this study was to compare these two prospective bacterial surveillance methods with regard to accuracy, efficiency, and cost.

\section{Methods}

The study was deemed as non-human subject research by the University of Chicago Institutional Review Board (IRB 19-0041) and did not require review.

At a single tertiary care center, 17 Olympus (Center Valley, PA) duodenoscopes TJF-Q180V series and 8 Olympus linear echoendoscopes GF-UCT180 series were included in the sample. The sample period ran from March 12, 2019, through August 16, 2019. After each use, following hospital and manufacturer guidelines for high-level disinfection and reprocessing, all elevator-containing endoscopes were placed in an automatic endoscope-drying machine. The automatic drying machine circulated filtered air through all the channels of the endoscope for at least 1 hour and 45 minutes. Upon removal from the machine, the endoscopes were removed and hung overnight in an upright position. All of these endoscopes were cultured for bacterial surveillance the following weekday morning. Endoscopes first underwent the brush protocol, followed immediately by the swab protocol. As the brush protocol was the organization's standard operating plan, the infection control department deemed it necessary to conduct this protocol first to avoid discrepancies in results if it was performed second. Both protocols were conducted by the same nurses. Any positive endoscopes were removed from circulation, reprocessed, and re-cultured until three consecutive negative cultures were obtained. Culture samples were sent to the microbiology laboratory for bacterial culture and susceptibility testing. This consisted of concentrating the samples and then using it to inoculate a solid and liquid bacteriological culture medium. Quantification was not performed, but identification and susceptibility testing were conducted on any Gram-negative bacilli that were isolated.

\section{Brush Protocol}

Supplies required for each culture included personal protective equipment, a sterile specimen trap and cup from Medline Industries (Northfield, IL), a suction canister and tubing, two 500-mL bottles of sterile water, a $\mathrm{CO}_{2}$ outlet connector, a Boston Scientific (Marlborough, MA) HedgeHog singleuse cleaning brush, and endoscope buttons (Table 1). All these supplies were gathered and prepared prior to culturing. The sterile cup was filled with $40 \mathrm{~mL}$ of sterile water from one $500-\mathrm{mL}$ sterile water bottle. From the same bottle, $360 \mathrm{~mL}$ of sterile water was discarded so that only $100 \mathrm{~mL}$ remained in the bottle. This sterile water bottle was used to hold all the water samples and brush sample. The other 500-mL sterile water bottle was attached to the $\mathrm{CO}_{2}$ outlet connector. The endoscope being cultured was plugged into the processor, and the $\mathrm{CO}_{2}$ outlet was connected to the endoscope. The suction canister was placed into the holder, connected to the suction tubing, which had the sterile specimen trap attached, and then, the trap was connected to the endoscope suction adapter.

Between two special procedure nurses, the brush protocol culture method was used to obtain the culture sample (Fig. 1). With the elevator mechanism in an up position, the $40 \mathrm{~mL}$ of sterile water is suctioned three times from the sterile cup while applying full suction, followed by suctioning three times with the elevator mechanism in a down position while applying half suction. The sterile water is emptied from the specimen trap back into the sterile cup so the same water sample is being suctioned each time. Next, $50 \mathrm{~mL}$ of sterile water is dispensed into the sterile cup while intermittently dispensing air. These two water samples are poured into the sterile water bottle with $100 \mathrm{~mL}$ of sterile water remaining. Using the big end of a single-use cleaning brush, both openings of the suction channel, the biopsy port, the suction adaptor, and above

Table 1 Supplies needed for culturing

\begin{tabular}{ll}
\hline Brush protocol & Swab protocol \\
\hline $\begin{array}{l}\text { Personal protective equipment } \\
\text { 40-mL sterile specimen trap }\end{array}$ & $\begin{array}{l}\text { Personal protective equipment } \\
\text { Flocked swab specimen collec- } \\
\text { tion kit }\end{array}$ \\
Suction canister and tubing & 10-cc sterile saline syringe \\
120-mL sterile cup & \\
500 -mL sterile water bottle (2) & \\
$\mathrm{CO}_{2}$ outlet connector & \\
Single-use cleaning brush & \\
Endoscope buttons &
\end{tabular}


Fig. 1 Brush protocol for elevator-containing endoscopes. Brush protocol for elevatorcontaining endoscopes. Sterile water is suctioned into the specimen trap $(\mathbf{a}, \mathbf{b})$. Sterile water is dispensed while intermittently dispensing air $(\mathbf{c}, \mathbf{d})$. Both openings of the suction channel (e, f), the biopsy port (g), the suction adaptor (h), and above and below the elevator mechanism $(\mathbf{i}, \mathbf{j})$ are brushed using the big brush. The small brush is passed through the suction channel out the suction adapter (k) and out of the distal tip of the endoscope (l)
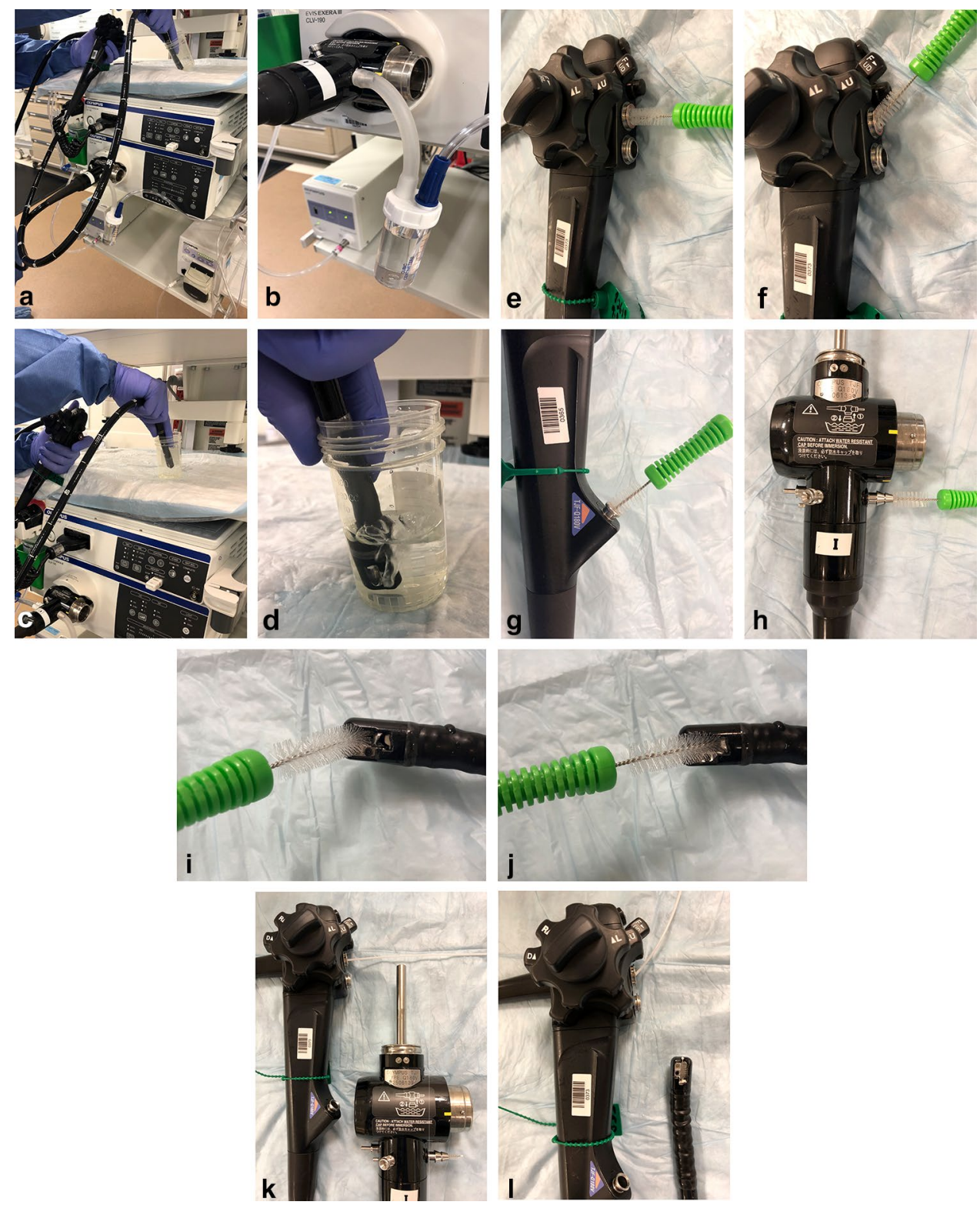

and below the elevator mechanism are brushed. The small end of the same single-use cleaning brush is then used to brush through the suction channel opening until the brush appears out of the suction adapter. The same end is then used to brush through the other suction channel opening until the brush appears out of the distal tip of the endoscope. After brushing through both channels, the brush is then placed into the same $100-\mathrm{mL}$ sterile water bottle that holds the water samples. The bottle is closed and sent to the microbiology laboratory for testing. All of the equipment, except for the $\mathrm{CO}_{2}$ outlet connector, used for this culture specimen is discarded, and a new set of supplies is required for each individual endoscope.

\section{Swab Protocol}

The swab protocol was conducted on each endoscope immediately following the conclusion of the brush protocol sampling process. Equipment required for each culture included personal protective equipment, a sterile saline syringe, and a Fisher Scientific (Waltham, MA) Copan Diagnostics ESwab flocked swab specimen collection kit, and was gathered prior to culturing (Table 1).

Between two special procedure nurses, the swab protocol was performed to gather the culture sample (Fig. 2). With the elevator mechanism in the mid-position, $1 \mathrm{~mL}$ of sterile saline was dripped onto the elevator mechanism. The flocked swab from the specimen collection kit was 
Fig. 2 Swab protocol for elevator-containing endoscopes. Sterile saline is dripped onto the elevator mechanism (a). The flocked swab is used to swab above and below the elevator (b, c). The swab is broken into the specimen tube (d)

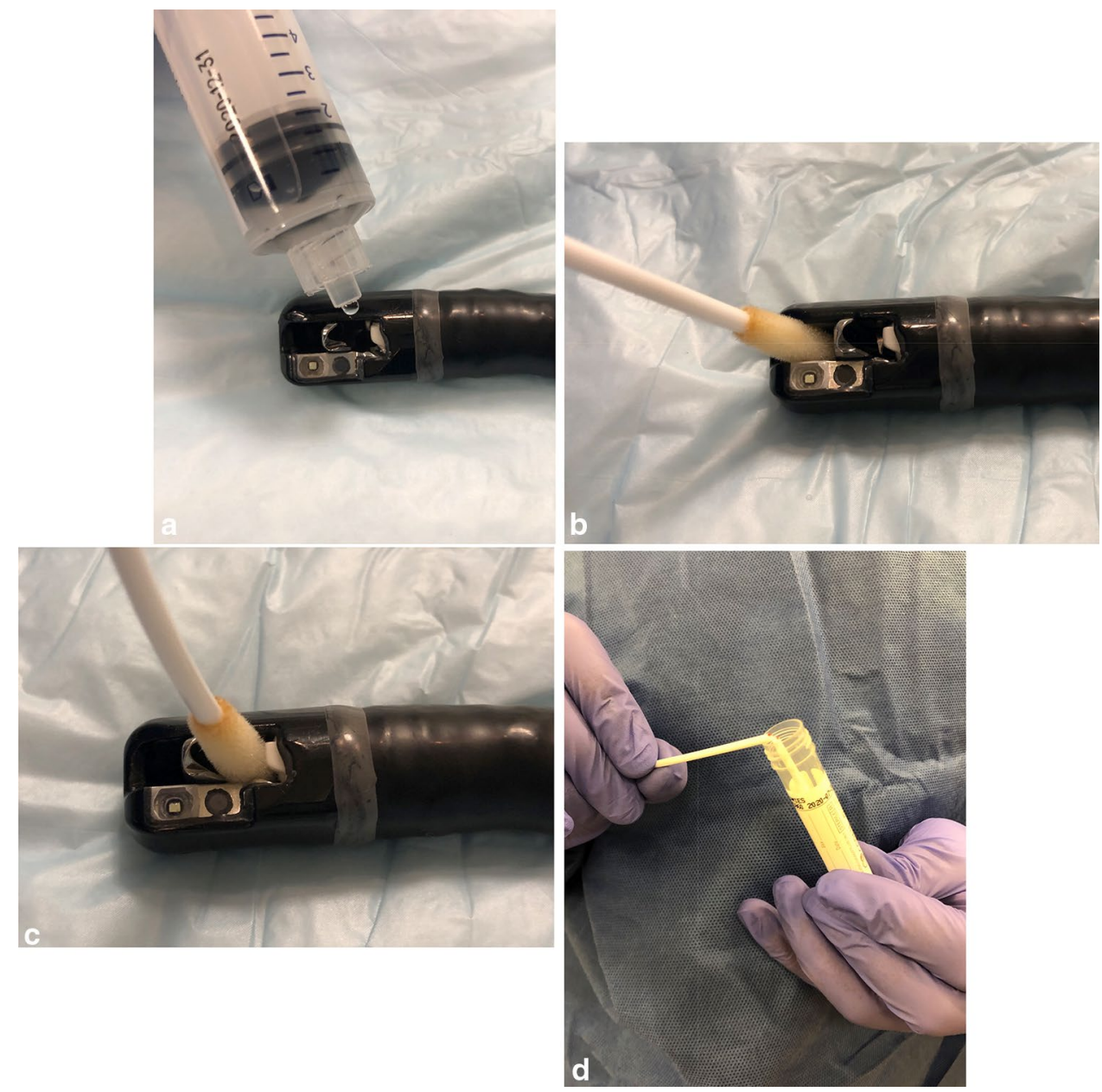

used to swab above and below the elevator mechanism. Next, the swab was broken off into the specimen tube from the collection kit, sealed, and sent to the microbiology laboratory for testing.

\section{Cost-Effectiveness Modeling}

Costs for each protocol were based on price per culture. Annual supply costs for the brush protocol were $\$ 5,527$, while the swab protocol costs were $\$ 1,913$. Calculations for nursing time were based on how long it took to obtain samples for each surveillance method and the time of two nurses for 5 days a week for 52 weeks. The nursing time cost was calculated based on the average hourly rate of $\$ 47.90$ per hour per nurse. More supplies (Table 1) and time are needed for the brush protocol, which accounts for the increased cost for the brush protocol compared to the swab protocol (Table 3 ).
Table 2 Brush and swab protocol culture results

\begin{tabular}{llll}
\hline & Negative & Positive & Defect rate (\%) \\
\hline Brush protocol & 266 & 0 & 0 \\
Swab protocol & 263 & 3 & 1.1 \\
\hline
\end{tabular}

Table 3 Annual cost for culturing

\begin{tabular}{lll}
\hline & \multicolumn{2}{l}{ Annual cost $(\$)$} \\
\cline { 2 - 3 } & Brush protocol & Swab protocol \\
\hline Culture testing & 35,892 & 35,892 \\
Nursing time & 24,908 & 1,987 \\
Supplies & 5,527 & 1,913 \\
Total & 66,327 & 39,792 \\
\hline
\end{tabular}

*Nursing time cost based on average rate of $\$ 47.90 /$ hour 


\section{Results}

A total of 532 primary cultures were collected from 17 duodenoscopes and eight linear echoendoscopes. Of these, 266 cultures gathered using the brush protocol were negative, while 266 cultures gathered using the swab protocol resulted in three positive cultures $(1.1 \%)$ (Table 2). Positive cultures came from one duodenoscope and two linear echoendoscopes and tested positive for Enterobacter cloacae, Klebsiella pneumoniae, and Escherichia coli that was a multi-drug-resistant organism with extended spectrum beta lactamase production.

The brush protocol took an average of $12 \mathrm{~min}$ to perform and cost approximately $\$ 53.40$ per culture, while the swab protocol took an average of $1 \mathrm{~min}$ and cost an estimated $\$ 32.04$ per culture. Using the cost-effectiveness modeling as stated earlier, the brush protocol would take an estimated 520 nursing hours per year and the swab protocol would take an estimated 42 nursing hours per year. Nursing hours were projected based on historical volumes, which required the culturing of 1242 endoscopes with an elevator mechanism. The brush protocol costs an estimated $\$ 66,327$ per year, while the swab protocol costs an estimated $\$ 39,792$ per year (Table 3 ). The swab protocol could potentially save over $\$ 26,500$ and 478 nursing hours annually.

\section{Discussion}

In this pilot study data, the swab protocol was superior to the brush protocol when evaluating the presence of residual bacterial found on duodenoscopes and linear echoendoscopes. The swab method had a higher detection rate, as it was able to detect three positive cultures not found using the brush protocol, including a multi-drug-resistant organism. Additionally, the endoscopes that tested positive were from one duodenoscope and two linear echoendoscopes, which supports the need to survey both elevatorcontaining endoscopes as shown in previous studies $[1,2]$. Furthermore, the swab method appears less susceptible to environmental contamination as the brush method poses a higher risk due to the increased duration of sampling [4]. Additionally, the flocked swab design allows for better sampling due to increased bioadhesion of microorganisms compared to the harder, cleaning bristles used in the brush protocol [5]. Based on these results, the proposed swab protocol can offer a more cost-saving, accurate, and efficient method for prospective bacterial surveillance for duodenoscopes and linear echoendoscopes.
Clinical relevance from this study includes a surveillance method to prevent endoscope-related bacterial outbreaks. As there continues to be a lack of standardized protocols, the proposed swab protocol has the potential to improve patient safety, as well as offer a more accurate and efficient method for identifying contaminated elevator-containing endoscopes. There are a few duodenoscope variations that have recently been proposed by manufacturers, and gained FDA approval, to obviate the potential contamination of elevators: completely disposable scopes, disposable caps on scope tip, and disposable elevators. However, it is unclear how widely available or cost-effective these alternatives will be, especially due to uncertainties related to the current COVID-19 pandemic. Currently, there are no plans for revising the linear echoendoscope design from its current iteration. Therefore, in lieu of future alternatives, it is necessary to utilize costeffective tools available right now in every endoscopy unit to ensure patient safety. The small sample size was one study limitation, and further prospective studies in larger sample sizes are needed to validate the optimal surveillance protocols for elevator-containing endoscopes. Risk for environmental contamination also poses a limitation to the utilization of the brush protocol for prospective bacterial surveillance. Strengths of using the flocked swab protocol include improved efficiency, a reduction in risk of contamination, increased bio-absorption of microorganisms [5], and cost-savings. With a lack of universal guidelines [3], a comparison of two approaches for prospective bacterial surveillance in elevator-containing endoscopes demonstrates the effectiveness of the novel flocked swab protocol.

\section{Compliance with Ethical Standards}

Conflict of interest The authors declare that they have no conflict of interest.

\section{References}

1. Muscarella LF. Risk of transmission of carbapenem-resistant Enterobaceriaceae and related "superbugs" during gastrointestinal endoscopy. Work J Gastrointest Endosc. 2014;6:457-475

2. Chapman CG, Siddiqui UD, Manzano M, Konda V et al. Risk of infection transmission in curvilinear array echoendoscopes: results of a prospective reprocessing and culture registry. Gastrointest Endosc. 2017;85:390-397

3. Centers for Disease Control and Prevention. Duodenoscope surveillance sampling and culturing: Reducing the risks of infection. https://www.cdc.gov/hai/organisms/cre/cre-duodenosco 
pe-surveillance-protocol.html. Updated November 1, 2019. Accessed 2 Dec 2019.

4. Paula H, Tribl B, Presterl E, Diab-El Schahawi M. Prospective microbiologic evaluation of the forceps elevator in closedchannel duodenoscopes after reprocessing. Am J Infect Control 2017;45:121-125

5. Gazdik MA, Coombs J, Burke JP, Lopansri BK. Comparison of two culture methods for use in assessing microbial contamination in duodenoscopes. J Clinical Micro. 2016;54:312-316
Publisher's Note Springer Nature remains neutral with regard to jurisdictional claims in published maps and institutional affiliations. 